\title{
MS13-01 | Bioinspired Calcium Phosphates: Structural Modifications Induced by
}

\section{FUNCTIONALIZATION}

Boanini, Elisa (University of Bologna, Bologna, ITA)

Thanks to their excellent biocompatibility and bioactivity, calcium orthophosphates are widely employed in the preparation of biomaterials for hard tissues substitution and repair. Improvement of tissue response can be achieved through the development of functionalized calcium phosphate based materials. Furthermore, tailormade multi-functionalized calcium phosphates may be exploited to provide local release of the functionalizing agents and prevent the drawbacks of systemic treatments. This lecture will focus on the role played by the structural approach on the comprehension of the important influence of functionalization with bioactive ions and molecules on the structure, morphology, and stability of some calcium orthophosphates of biological interest. 